\title{
Research on the Promoting Effect of Tai Chi Exercise on Improving Physical Health in Fitness Activities
}

\section{Lin Wang, and Weifeng Deng}

\author{
Hainan Vocational University of Science and Technology Haikou, Hainan 571126, China
}

893094362@qq.com

Keywords: Tai Chi exercise, fitness activities, healthy living.

\begin{abstract}
Physical health is the eternal theme of human pursuit. Among the many fitness exercises, Tai Chi exercises are slow and soft, emphasizing awareness, movement and breathing coordination, and the balanced development of the human body. It is suitable for health preservation, healing and extension of different groups of people. Years of needs. This article analyzes the scientific basis of Tai Chi exercise and analyzes its performance characteristics and fitness effects.
\end{abstract}

\section{Introduction}

Tai Chi is a traditional sports event in our country, and it is our country's precious cultural heritage. It has a history of nearly 400 years. Tai Chi soft ball exercise can not only exercise the body, but also enjoy the body and mind. It is a sport that combines happiness in physical exercise. It is not restricted by age, gender, physique or physical fitness. The technology is simple and easy to learn. The number of participants, venue, and equipment It can be determined according to the actual situation. It is a sport that everyone can participate in [1]. Once it was launched, it has been loved by the majority of middle-aged and elderly people. Now the majority of young people are actively participating in this sport. The author believes that the promotion and popularization of Tai Chi softball in our country can not only better promote the development of national fitness activities and increase the number of sports populations in our country, but also enable traditional Chinese sports to be inherited and developed. It is believed that Tai Chi soft ball will occupy an important position and play an active role in national fitness activities with its unique charm and project advantages.

\section{The value of Tai Chi in national fitness}

\subsection{Social value}

The rise and fall of a country, the progress of society, and the development of the economy all depend on people and their quality. The physical quality of workers is the material basis of ideological and moral quality and scientific and cultural quality. With the advancing of the times, society is required to be more civilized and human beings are healthier and stronger [2]. The rise of nationwide fitness undoubtedly provides the possibility and guarantee for the progress of society and the progress of mankind. As a traditional sports event in my country, Tai Chi sports integrates Chinese traditional cultural ideas, views and values such as philosophy, medicine, and aesthetics. It has a strong Chinese traditional cultural colour in terms of content, sports forms, practice methods, and ethics. Accepted and loved by the people.

\subsection{Fitness and health value}

Tai Chi exercises have distinct characteristics. When practicing, they are required to relax the mind, coordinate up and down, lead the body with the mind, guide the qi with the mind, and push the strength with the qi. Long-term exercise can clear the meridians, regulate qi and blood, and balance yin and yang, which is conducive to the recovery and improvement of brain function and enhances the body's function. In today's society, people's lives are tense, fast-paced, and mentally 
stressed. The number of patients with various diseases is increasing, which has formed a serious social problem. Through the implementation of the National Fitness Program, people gradually pay attention to physical exercise, and often participate in sports such as jogging, basketball, badminton, swimming, aerobics, tennis and other sports to relieve physical and mental fatigue, but these sports are not only compared with Tai Chi [3]. The restrictions on time, venue, equipment, age, climate, etc. are much greater, and they are not as good as Tai Chi which makes people relax physically and mentally, especially the brain. Because Tai Chi requires calmness, relaxation, and unity of mind and body, that is, Tai Chi not only has the exercise effect produced by these items, but also has greater medical and health value, and brings comfort and joy to people's body and mind.

\subsection{Economic and cultural value}

Tai Chi movement has received widespread attention from the international community and has produced a strong response. Various types of Tai Chi associations and clubs at various levels at home and abroad have sprung up, and the teams participating in the Tai Chi movement continue to grow and grow. Sports set up the stage, economic performance. With the development of various types of Tai Chi competitions and exchanges at all levels, it will surely promote the development of domestic tourism, trade, culture and other industries, and generate considerable economic benefits. Moreover, it can promote the Tai Chi movement, the excellent traditional culture of the Chinese nation, to the world and carry it forward.

\section{Tai Chi Kinematics Data Capture}

\subsection{Kinematics modelling based on somatosensory technology}

The completion of various actions of the human body has its own complexity and regularity. Any simple action is controlled by many internal systems in the process of its completion. Reflected in sports, each set of actions completed by athletes is also affected by multiple systems of the human body. The current kinematics research also mainly analyzes its regularity from the perspective of time and space. Therefore, the scientific nature of the analysis of human kinematics can be reflected only after fully considering the reference indicators such as the track and recorded speed left by the athletes during the exercise.

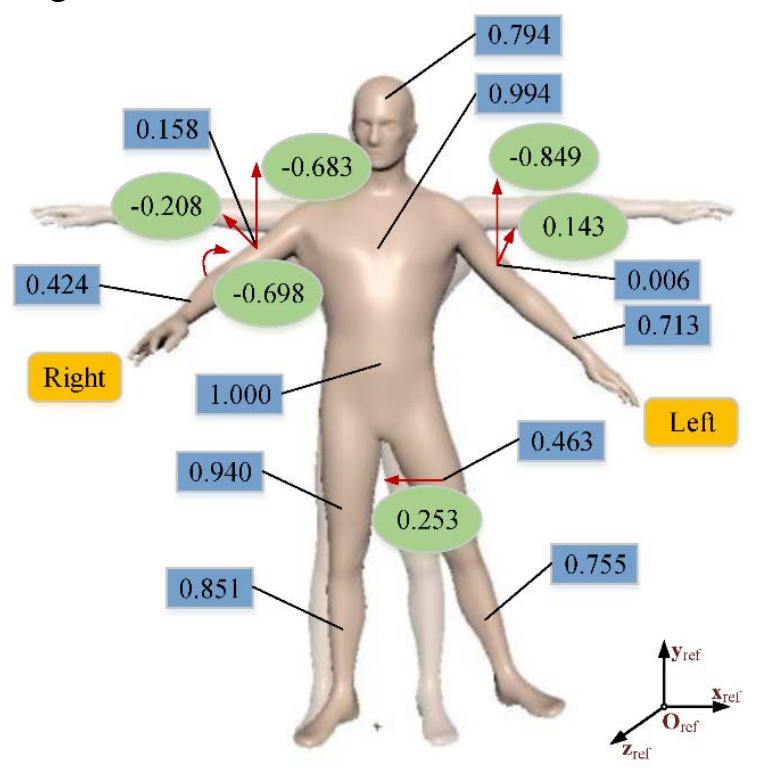

Figure 1. Spatial coordinate system of data flow

We know that kinematics only discusses the movement itself, and does not involve the internal and external forces that produce the movement, so we think that the kinematics-based model means that the movement of the object is independent of the trajectory and spatial position of the movement, and its parameters mainly include the spatial position of the object, Speed and 
acceleration. It is not difficult to conclude that the complete kinematic parameters of the human bone segment can be described as the parameters of the time change, including the position of the centre of gravity of the human bone segment, the angle of the human bone segment, the acceleration of the human bone segment, the core velocity of the human bone segment, and the human body The angular velocity of the bone segment, the body centre acceleration of the human bone segment, etc. In order to reduce the complexity of the research, when we describe the specific motion behaviour, we make reasonable simplifications, and use a small part of many motion parameters to complete the description of this motion. In most cases, the collected raw coordinate data cannot be used directly. The measurement methods for bone point data and depth data, including colour image data, are different. Each type of data (image data, bone data, depth data) is defined in a specific set of coordinates or space; generally, depth data or the image data is represented by pixels, and the $\mathrm{X}$ and $\mathrm{Y}$ positions start at 0 from the upper left corner. The $\mathrm{Z}$ position data of the depth data is in millimetres. As shown in Figure 1.

\subsection{Tai Chi kinematics data model capture}

Motion capture is a multifaceted target, including positioning measurement, physical space positioning, etc. measurement, and directly integrated data processing technology by a computer; it sets tracker nodes on the key parts of the moving object, and the motion capture system captures and tracks the position of the device is processed by the computer to give feedback to the user. When the computer receives the data collected by us, the user's movement trajectory in front of the lens will be recorded by the computer, and the generated image will be displayed on the monitor in real time or research based on relevant data. The virtual reality motion capture system is a technology that records human motion information for analysis and playback. The captured data can not only be as simple as recording the spatial position of the body parts, but also as complex as recording the detailed movements of muscle groups and faces; and the motion capture applied to computer character animation involves how to convert real human actions into digital models [4]. This conversion mapping can be direct, like using a real subject's arm movements to control the arm movements of a digital actor; it can also be indirect, like using a real subject's arm and finger movements to control the skin colour of a digital model and emotions.

The somatosensory game of Tai Chi theme can analyse the wrong postures and movements in many actions. In the research, we applied an interactive video reflection motion analysis system, based on the KINECT technology, using the settings shown in Figure 2. Its advantage is that it can repeatedly perform and evaluate the standardization of Tai Chi movements. For example, by delaying the camera view for a few seconds, you can perform a spin kick and immediately see the repetitive action on the screen, which is more convenient than traditional capture devices. The physical interaction area is fixed and will not change with the change of the Kinect area (such as horizontal and vertical). This area should be fully considered when collecting, and the Kinect area should be designed according to this to ensure that users can be more accurate and comfortable operating.

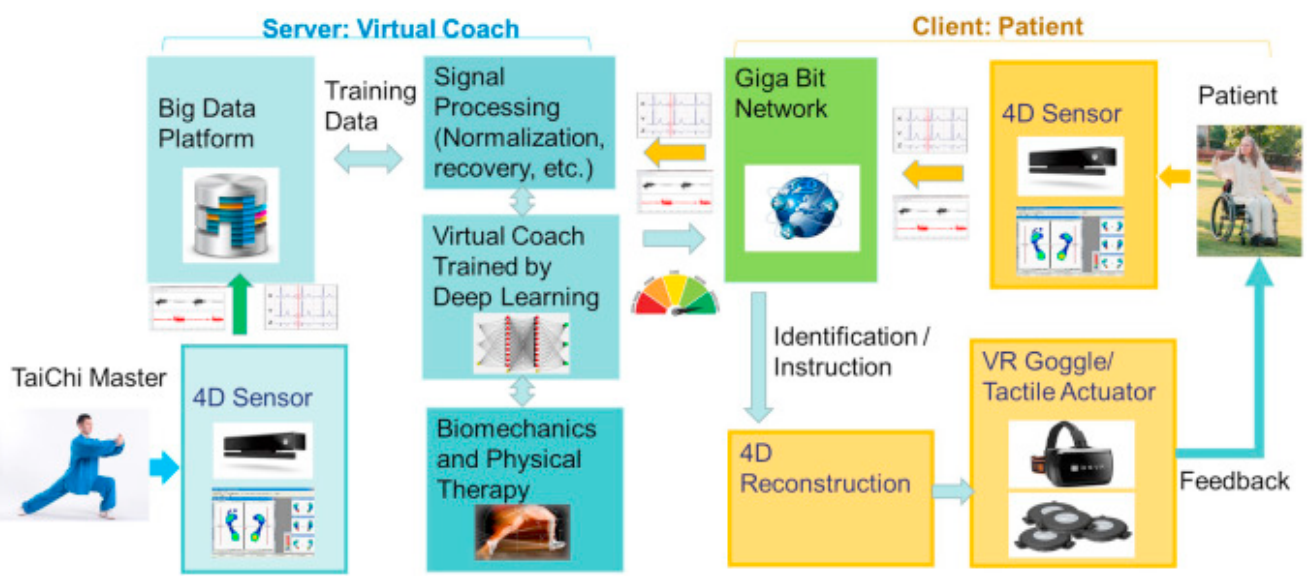

Figure 2. Tai Chi sports video mirror test setup 


\subsection{Self-encoding network structure and training method}

The network proposed in this paper consists of two parts: an encoder and a decoder, as shown in Figure 3. The encoder is composed of a 3-layer fully connected network (FC) and a two-way long and short-term memory network (Bi-LSTM). The first layer The number of neural units in FC is 128 , the number of neural units in the second layer of FC is 256, and the number of neural units in the third layer of FC is 512; the current dimension of the input data is $\mathrm{m} \square 512$, and $\mathrm{m}$ is the number of frames of motion data initially input to the encoder ; Bi-LSTM output data dimensions are the same; the output of the encoder is recorded as a hidden variable, and the decoder structure is symmetrical to the encoder.

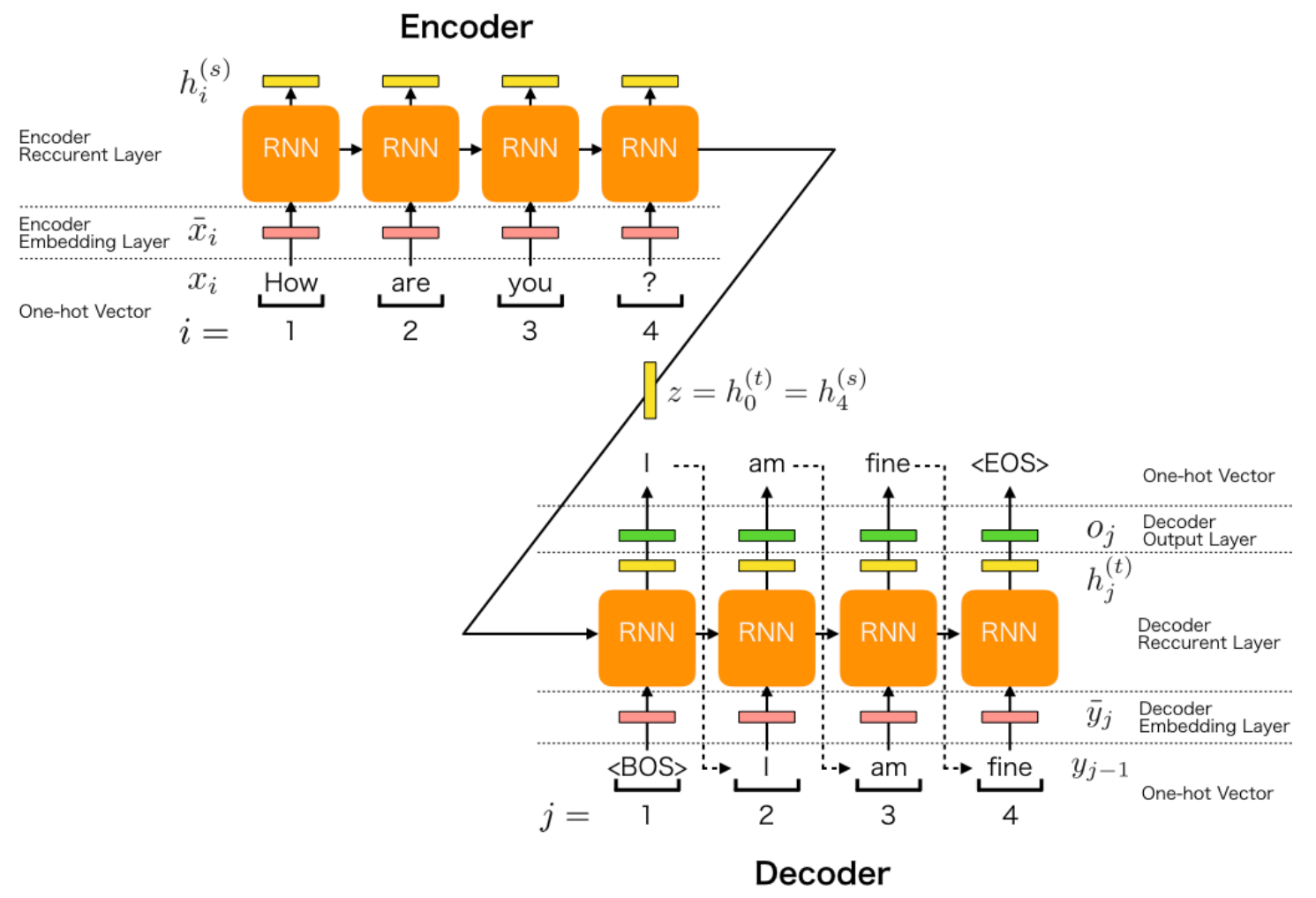

Figure 3. Self-encoding network structure and loop iteration method

The input motion segment used in this article when training the network is 120 frames in length, that is, the network input is a matrix of $120 \times 63$, denoted as $X_{i j}$. The motion data $X_{i j}$ passes through the encoding network, extracts the data features and saves it to the hidden unit, and the hidden unit data is input to the decoding network output Rebuild the motion $\mathrm{Y}$, repeat the above process many times to train the autoencoder. The loss function of network training is the reconstruction error, and the expression is

$$
L=\frac{1}{m \times n}\left\|X_{i j}-D_{\varphi}\left[E_{\varphi}\left(X_{i j}\right)\right]\right\|_{2}
$$

Among them, $E_{\varphi}\left(X_{i j}\right)$ represents the hidden unit data obtained by the motion data through the encoder; $D_{\varphi}\left[E_{\varphi}\left(X_{i j}\right)\right]$ represents the hidden unit data after the decoder; $\mathrm{m}$ and $\mathrm{n}$ are the number of rows and columns of the matrix $X_{i j}$. The idea of this article is to optimize the properties of Y to hide Edit $\mathrm{H}$ in the variable space, and finally get the motion data of the target character. That is, calculate the attribute loss function of the decoder output data Y, backpropagate to the hidden layer, and then update the hidden variable $\mathrm{H}$ through the optimization algorithm iteratively to minimize the loss function Finally, the optimal hidden variable $\mathrm{H} \square$ is obtained to satisfy

$$
H^{\prime}=\arg \boldsymbol{m i n}\left(\lambda_{\text {bone }} L_{\text {bone }}\left(D_{\varphi} H\right)\right)+\lambda_{\text {foot }} L_{\text {foot }}\left(D_{\varphi} H\right)+\lambda_{\text {root }} L_{\text {root }}\left(D_{\varphi} H\right)
$$


Among them, the role of $L_{\text {bone }}$ is to make the redirection motion meet the bone length requirements; the role of $L_{\text {foot }}$ is to make the redirection motion meet the footprint constraint; the role of $L_{\text {root }}$ is to make the redirection motion and the root joint position of the source motion meet the proportional relationship.

\section{The effect of Tai Chi on physical health}

Tai Chi movements are gentle and emphasize abdominal breathing. It mainly promotes thoracic and lung movement through the lifting and lowering of the diaphragm muscles, increasing the depth of breathing, not only massages the internal organs, but also promotes the reduction of airway resistance, the increase of lung flux, and the promotion of body metabolism. Long-term practice of Tai Chi can enhance left ventricular myocardial contractility, improve heart pumping function, improve myocardial diastolic capacity, increase cardiac output per beat and per minute output, reduce heart rate in a quiet state, and improve and improve cardiac function. Medical practice has proved that regular practice of Tai Chi has a good therapeutic effect on heart disease, premature beats, palpitations, tachycardia and respiratory diseases, as well as relieving and inhibiting hypertension. High blood pressure is mostly triggered by mental factors. Mental tension and bad mood can cause qi and blood to surge, dizziness and upset, and blood pressure rises quickly [5]. Tai Chi practice requires loose body meditation and Qi sinking in Dantean. The brain is refreshed if the qi is heavy, and the blood pressure is stable if the qi and blood are unblocked. Tai Chi exercises are gentle and long, so they will not increase the burden on the heart like some other exercises that are too intense and intense, and are not forbidden by doctors.

Neurasthenia is a disease caused by excessive excitement of the brain, inability to suppress itself, and frequent insomnia. It is medically called autonomic disorder. The pressure of modern people's life and work often causes the corresponding areas of the cerebral cortex to be in a state of high excitement for a long time, which can easily lead to the overexcitement of the sympathetic nerves, while the excitability of the parasympathetic nerves is relatively reduced, and the two are in a state of imbalance. Tai Chi exercise emphasizes calmness and naturalness, requires calmness and emptiness, and forgets things. The concentration on training shows that athletes' performance declines and the training plan needs to be adjusted in time. Therefore, urobilinogen is also one of the important indexes to evaluate the physical function of middle- and long-distance runners.

\section{Conclusion}

Tai Chi soft ball sport has the connotation and philosophy of oriental culture, which integrates tradition and modernity, and integrates fitness, performance and competition. Tai Chi soft ball is a high fitness value, simple, easy, time-saving, labour-saving, money-saving and convenient for a wide range of exercises. It must be able to play a very important role in national fitness activities.

\section{Acknowledgments}

[Foundation project] 2019 Hainan Provincial Higher Education Scientific Research Project (HnKy2019-104)

\section{References}

[1] Wang, Y. T., Taylor, L., Pearl, M., \& Chang, L. S. Effects of Tai Chi exercise on physical and mental health of college students. The American Journal of Chinese Medicine, 32(03) (2004) 453459.

[2] Thornton, E. W., Sykes, K. S., \& Tang, W. K. Health benefits of Tai Chi exercise: improved balance and blood pressure in middle-aged women. Health promotion international, 19(1) (2004) 33-38. 
[3] Lam, L. C., Chau, R. C., Wong, B. M., Fung, A. W., Lui, V. W., Tam, C. C., ... \& Chan, W. M. Interim follow-up of a randomized controlled trial comparing Chinese style mind body (Tai Chi) and stretching exercises on cognitive function in subjects at risk of progressive cognitive decline. International journal of geriatric psychiatry, 26(7) (2011) 733-740.

[4] Taylor-Piliae, R. E., Haskell, W. L., Waters, C. M., \& Froelicher, E. S. Change in perceived psychosocial status following a 12-week Tai Chi exercise programme. Journal of advanced nursing, 54(3) (2006) 313-329.

[5] Taylor-Piliae, R. E., Newell, K. A., Cherin, R., Lee, M. J., King, A. C., \& Haskell, W. L. Effects of Tai Chi and Western exercise on physical and cognitive functioning in healthy communitydwelling older adults. Journal of aging and physical activity, 18(3) (2010) 261-279. 